\title{
Neuroanatomy of the middle cerebral artery: implications for thrombectomy
}

\author{
Maksim Shapiro, ${ }^{1}$ Eytan Raz, ${ }^{2}$ Erez Nossek, ${ }^{3}$ Breehan Chancellor, ${ }^{1}$ Koto Ishida, ${ }^{4}$ \\ Peter Kim Nelson ${ }^{1}$
}

${ }^{1}$ Radiology and Neurology, New York University Langone Medical Center, New York, New York, USA

${ }^{2}$ Radiology, NYU Langone Medical Center, New York, New York, USA

${ }^{3}$ Neurosurgery, NYU School of Medicine, New York, New York, USA

${ }^{4}$ Neurology, New York University Langone Medical Center, New York, New York, USA

\section{Correspondence to} Dr Maksim Shapiro, Radiology and Neurology, New York University Langone Medical Center, New York, NY 10016, USA; maksim.shapiro@nyumc. org

Received 31 December 2019 Accepted 27 January 2020 Published Online First 27 February 2020
Check for updates

(C) Author(s) (or their employer(s)) 2020. No commercial re-use. See rights and permissions. Published by BMJ.

To cite: Shapiro M, Raz E, Nossek $E_{\text {, et }}$ al.

J Neurolntervent Surg

2020:12:768-773.

\section{ABSTRACT}

Our perspective on anatomy frequently depends on how this anatomy is utilized in clinical practice, and by which methods knowledge is acquired. The thrombectomy revolution, of which the middle cerebral artery (MCA) is the most common target, is an example of a clinical paradigm shift with a unique perspective on cerebrovascular anatomy. This article reviews important features of MCA anatomy in the context of thrombectomy. Recognizing that variation, frequently explained by evolutionary concepts, is the rule when it comes to branching pattern, vessel morphology, territory, or collateral potential is key to successful thrombectomy strategy.

\section{CLINICALLY RELEVANT PHYLOGENY AND EMBRYOLOGY}

The middle cerebral artery (MCA) is the phylogenetically youngest cerebral vessel. In "lower" species, primarily allocortical structures-such as olfactory and hippocampal areas-are supplied by anterior cerebral artery (ACA) and posterior cerebral artery (PCA)-like vessels, respectively, while basal ganglia structures are served by "perforating" branches of the proximal "cranial ramus" or ACA homologs. Expansion of the neocortex in progressively "higher" species is accompanied by recruitment of vessels from the existing lenticulostriate system to supply the neocortical mantle. Thus, from an evolutionary perspective, the MCA is both a branch of the ACA and a branch of lenticulostriate vessels, not vice versa. ${ }^{1}$ Simply put, MCA or MCAs can arise from anywhere lenticulostriates do. This perspective helps explain various accessory/duplicated MCA configurations, fenestrations, as well as variations of lenticulostriate anatomy.

\section{Lenticulostriate vessels}

The lenticulostriate vessels are a contiguous family, with origins along the traditional A2, A1, M1 and M2 segments. Subdividing them into "medial" or "lateral" subgroups, or assigning special significance to the artery of Heubner just because it is an eponym (it is simply the most medial of lenticulostriates and frequently arises from the proximal A2 segment ${ }^{23}$ ) is helpful from a descriptive standpoint; however, it is a functionally artificial concept that detracts from our appreciation of how the family functions as a whole. As in any balanced system, a spectrum of variation exists, leading to either common or separate origins of individual perforator vessels from either the ACA or MCA and the relative dominance of more medial versus more lateral trunks (figure 1). A more dominant "Heubner" will pick up the classical "medial lenticulostriate" territory of the proximal M1, while at the other end a distinct "Heubner" may not be identifiable because vessels supplying its territory happen to originate from the "medial lenticulostriate" group of the $\mathrm{MCA}^{1}$ (figure 1).

\section{Accessory MCA/duplicated MCA}

About 1\% of the time, two MCA-like vessels are present. Distinction between "accessory MCA" arising from the ACA, and "duplicated MCA" arising from the distal ICA-with main classifications by $\mathrm{Teal}^{4}$ and Manelfe ${ }^{5}$ - are morphologically useful, but have no apparent basis in embryology. All are expressions of the phylogenetic origin of the MCA from the lenticulostriate system. The MCA is a hypertrophied lenticulostriate vessel that captures cortical territory. A duplicated/accessory MCA disposition simply reflects persistence of two separate vessels responsible for cortical supplyconceptually similar to "duplicated" anterior inferior or superior cerebellar arteries. Any place that "gives rise" to lenticulostriate vessels can be a site of MCA-like vessel origin. Figures 2 and 3 show the continuum between "duplicated" and "accessory" MCAs, demonstrating that this distinction may simply be a consequence of arbitrarily defining the location of the carotid terminus. ${ }^{6}$ (figures 2-4)

From a practical perspective, when faced with two MCA-like vessels, key questions are: what constitutes the territory of each trunk, and from where do the lenticulostriates arise? It is not true that only one trunk gives rise to lenticulostriates, particularly when a Heubner-like accessory MCA is present. Typically, accessory MCAs with origins along the ACA will tend to supply the superior division frontal territory, whereas in classical "duplicated MCAs" the more proximal vessel usually supplies the anterior temporal territory ${ }^{7}$ (figure 4).

The very rarely seen proximal MCA fenestration might be relevant in thrombectomy as either a point of occlusion or as a site of branch origin-particularly the anterior temporal branch which seems to originate frequently from one fenestration limb. ${ }^{8}$

\section{MCA trunk and branching patterns}

Here is the classic story: M1 bifurcates into the superior and inferior division M2 segments. One M2 is usually larger than the other (figures 5-10). The 


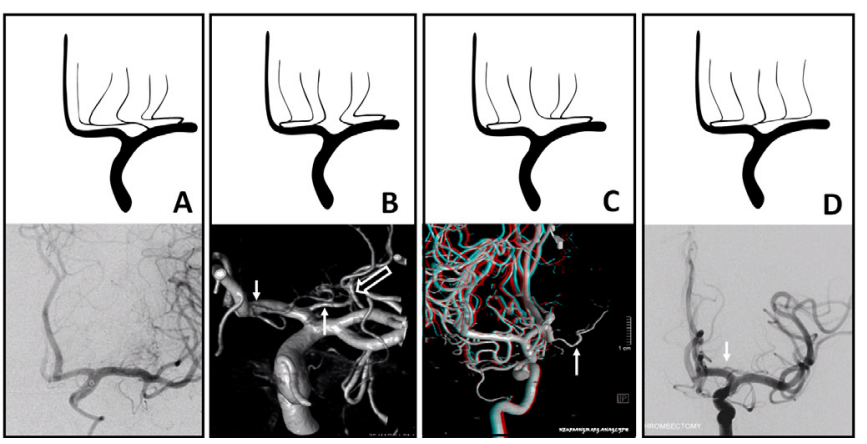

Figure 1 Schematic and comparable in vivo variations of lenticulostriate anatomy. The row of lenticulostriate perforators is a continuum of balance. Subdividing them into medial and lateral lenticulostriates is anatomically arbitrary. The Heubner (simply the most medial of perforators) may be absent or small (A). A1 perforators can have separate origins ( $B$, arrows) or arise as a common trunk (C). In extreme, a highly dominant $A 1$ system ( $C, D$ - white arrows) takes over the typical middle cerebral artery (MCA) origin lenticulostriates. Classical dominance of the MCA origin lenticulostriates is seen in (A). When short " $\mathrm{M} 1$ " trunks are present, more lateral perforators typically arise from the "M2" segment ( $B$, open arrow).

superior division supplies the frontal lobe, the inferior division supplies the temporal lobe. The parietal lobe is up for grabswhichever division supplies it is the "dominant" one. ${ }^{9}$ Typically,
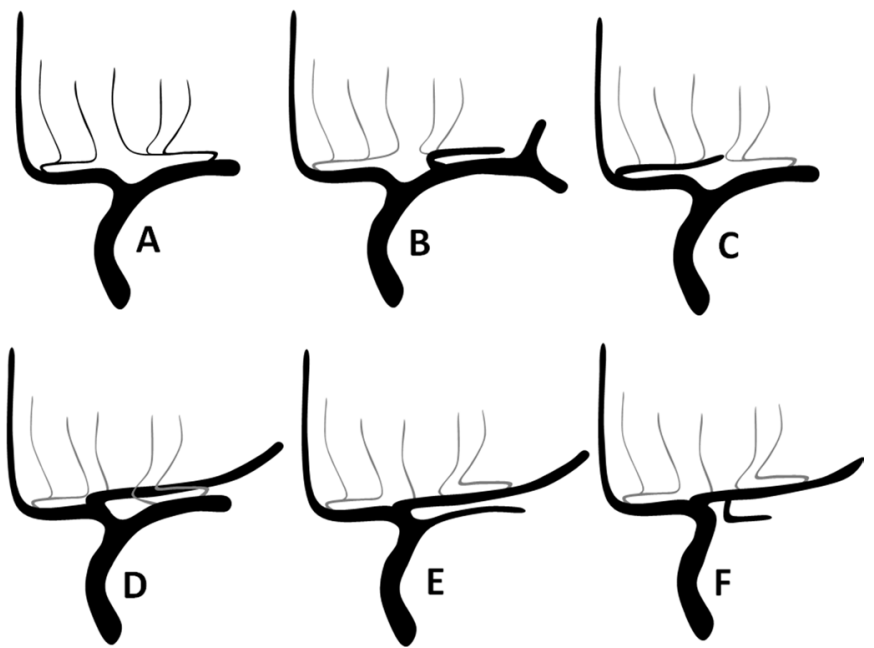

Figure 2 Schematic of accessory/duplicated middle cerebral artery (MCA) variations, underscoring a continuum based on the phylogenetic notion that MCA represents a hypertrophied lenticulostriate origin vessel serving neocortical territory. It can thus arise anywhere lenticulostriates come from. $(A)$ is classical disposition. (B) is a frequently unrecognized variant origin of the usually frontal branch from the "M1" segment lateral perforators, analogous to the more unusual origin of the same branch from the A1 segment (C) - appropriately regarded as a hypertrophied artery of Heubner, and commonly referred to as "accessory MCA". Criticism that this is not so because a separate Heubner-like artery may be present (D) ignores the simple fact that lenticulostriate vessels can have separate or common origins. Finally, (E) shows dominance of the proximal "A1" origin MCA vessel, with the smaller caliber "duplicated" MCA seemingly arising from the internal carotid artery (ICA). In fact, this is simply based on arbitrarily deciding where the ICA ends and A1 begins. (F) shows the early origin of the anterior temporal branch, a disposition that is contiguous with (E).
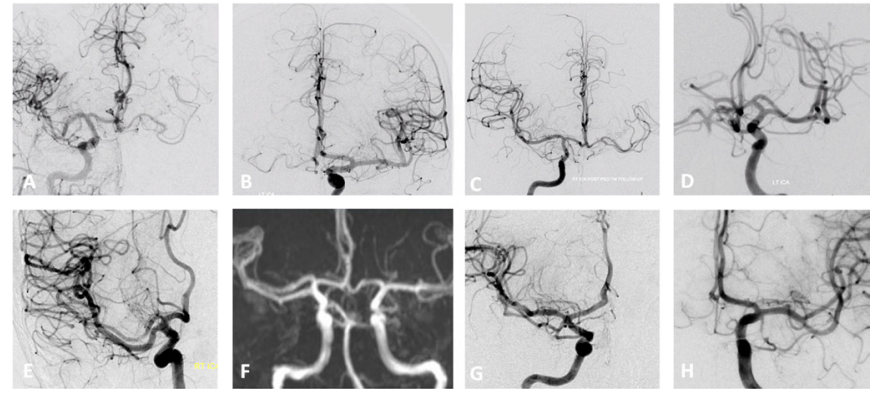

Figure 3 Spectrum of duplicated/accessory middle cerebral artery (MCA) vessels, from ACOM region Heubner-like variants $(A, B)$ to the mid-A1 origin ( $C, D$ - same patient, right and left internal carotid artery (ICA) injections), to the more proximal " $A 1$ " site (E,F), to "ICA terminus"-like disposition (G), to what is termed "duplicated" MCA (H). Which is "A1" origin and which is "ICA" origin is really based on an arbitrary notion of what represents the ICA terminus. In most cases, the "A1" origin MCA-like vessels supply the frontal territory, whereas the smaller vessels "arising" from the ICA $(\mathrm{G}, \mathrm{H})$ supply the anterior temporal lobe.

a small anterior temporal branch arises somewhere along the M1, before the "true" bifurcation. ${ }^{10}$ "Trifurcation", with each branch dedicated to frontal, parietal, and temporal lobes, is seen
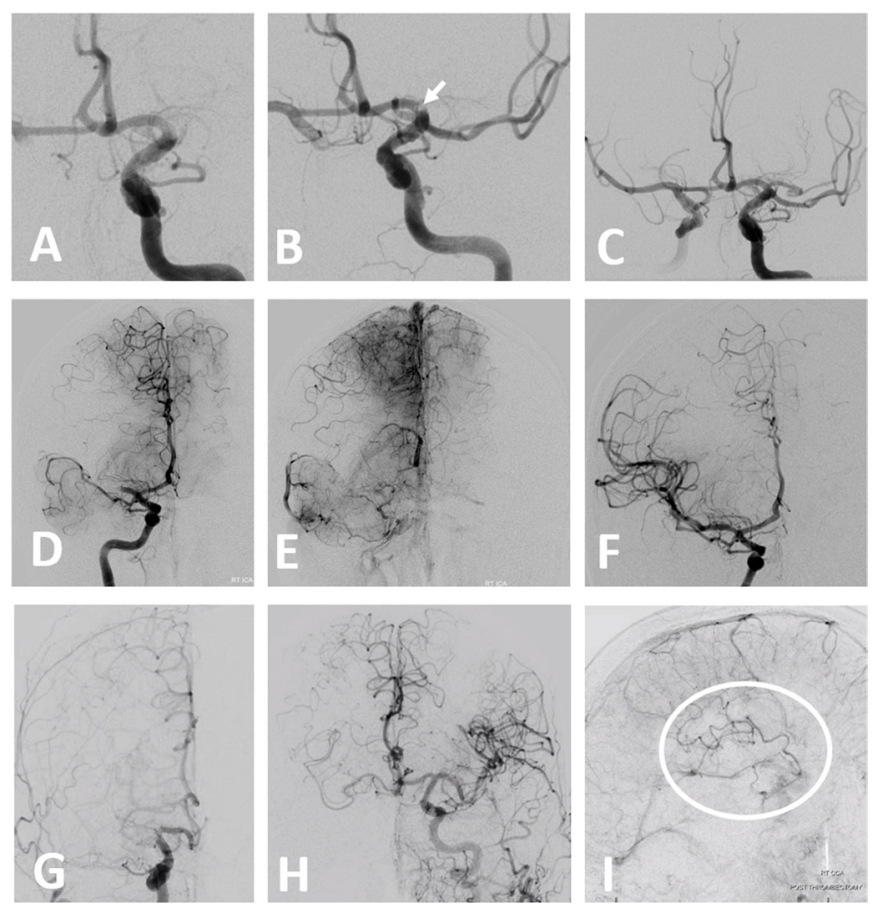

Figure 4 (A-F) Examples of dual middle cerebral artery (MCA) thrombectomy. Case 1, courtesy of Dr Daniel Sahlein $(A, B, C)$ : initial frontal view $(A)$, post-recanalization of dominant inferior division $(B)-$ arrow points to separate embolus in the still closed superior divisionand final recanalization (C). Case $2(D, E, F)$ : "duplicated" MCA supplying portions of temporal lobe. Embolus lodges in the larger branch that follows the vector of internal carotid artery (ICA) flow and supplies the bulk of the MCA territory, with partial anterior cerebral artery (ACA)MCA leptomeningeal collateral support (E). Lenticulostriate arteries arise proximal to the occlusion. Post-reperfusion (F). Case $3(\mathrm{G}, \mathrm{H}, \mathrm{I})$ : carotid terminus occlusion. Frontal convexity (white oval) remains perfused in an antegrade manner by the accessory MCA arising from the ACOM region $(H, I)$. 

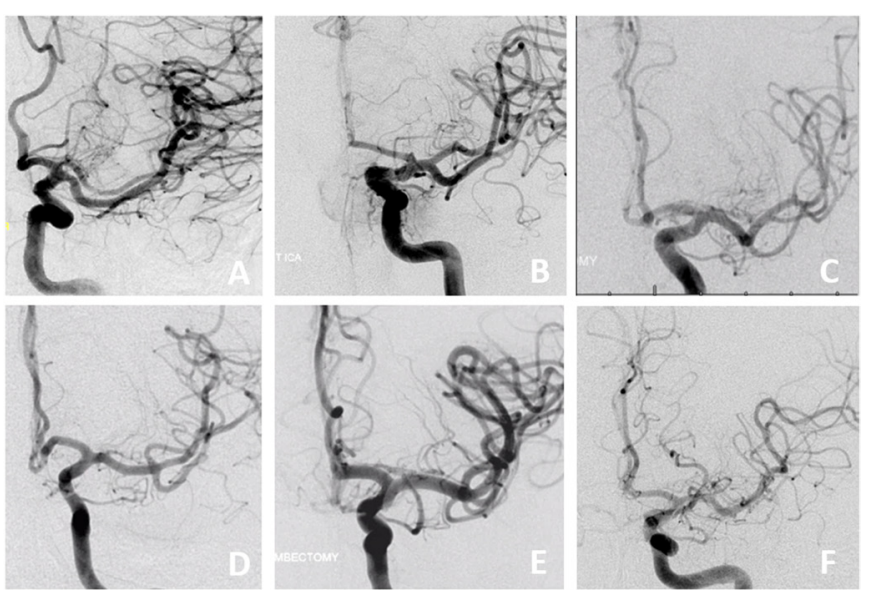

Figure 5 Spectrum of proximal middle cerebral artery (MCA) branching patterns. (A) Duplicated/accessory MCA pattern is contiguous with a "short M1" disposition (B), which is contiguous with a "classic" pattern (C) of M1 bifurcation into dominant and non-dominant M2s and any other conceivable variation, including "trifurcation" (D), "pentafurcation" (E), or no bifurcation at all (F), with vessels arising individually from a single common trunk.

in $\sim 10 \% .^{9-11}$ It is important to realize that life frequently does not follow rules (figure 8), and be ready for anything when we cannot see what lies beyond the M1 stump.

The length of the main MCA trunk is as variable as everything else about the MCA (figure 5). At one end of the spectrum there is no true MCA "bifurcation" or "trifurcation", with cortical vessels arising individually from the main trunk. When bifurcation or trifurcation is identified, the consensus is to call the main trunk "M1 segment". Short M1 segments blend into the "duplicated MCA" variant, underscoring limitations of defining what "M1" really is (figure $5 \mathrm{~A}, \mathrm{~B}$ ).

In regard to thrombectomy, the major dilemma is deciding where M1 ends and M2 begins. ${ }^{12} 13$ This definition can impact trials and potentially lead to exclusion of acute stroke patients from beneficial thrombectomy in less progressive centers. As boundaries of thrombectomy are extended into "M3" and "M4" segments, definitions become murkier still.

The surgical nomenclature of Gibo $e t a l^{9}$ of the M1 segment as extending horizontally along the sphenoid wing, M2 vertically along the operculum, M3 horizontally in the Sylvian fissure, and M4 on the surface of convexity was not designed to suit endovascular goals. Everything about MCA branching anatomy is summarized by "variability", hampering development of practical classifications based on variable branching patterns. ${ }^{12} 14$ The majority of studies addressing "M2" thrombectomy do not differentiate which side, division or territory was subject to occlusion, ${ }^{14-16}$ raising the question of selection bias. This subject was investigated in a retrospective single-institution study by Seker $e t$ $a l,{ }^{17}$ concluding that superior division occlusions, regardless of side, size or dominance, were independently predictive of poorer outcome compared with inferior division ones. Unsurprisingly, the National Institutes of Health Stroke Scale (NIHSS) also carries predictive value in M2 occlusions. ${ }^{18}$ For more distal (M3, M4) occlusions, very little data are available to guide thrombectomy decisions ${ }^{19}$; in all likelihood the bulk of "distal"-M3 and M4-interventions currently involves patients ineligible for intravenous treatments (figure 9).

Endovascular classifications should be based on supply territory rather than non-vascular landmarks or sites of vessel origin. Thus, the only thrombectomy classification that makes
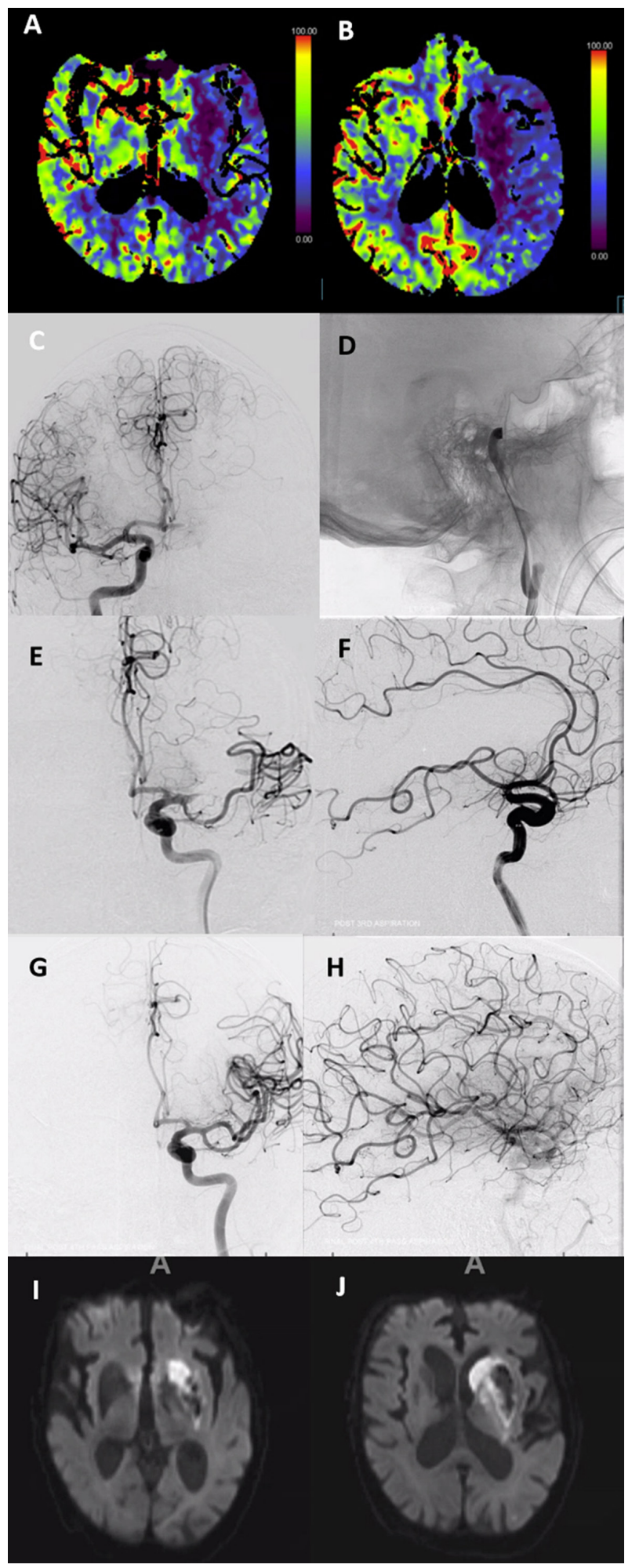

Figure 6 Analysis of carotid terminus occlusion. Loss of anterior choroidal and lenticulostriate territory is evident on perfusion imaging $(A, B)$, including caudate territory of the Heubner. From these two perfusion images alone we can deduce that the embolus is likely located below the choroidal artery, extends into the middle cerebral artery (MCA), and that there is no A1 segment origin of the Heubner, with its territory being served by occluded MCA lenticulostriates. Prethrombectomy $(C, D)$, after partial recanalization $(E, F)$ with dominant inferior division supplying frontal and parietal areas still closed, and full recanalization $(G, H)$. Delayed MRI $(I, J)$ shows excellent correlation with perfusion imaging in this case. 

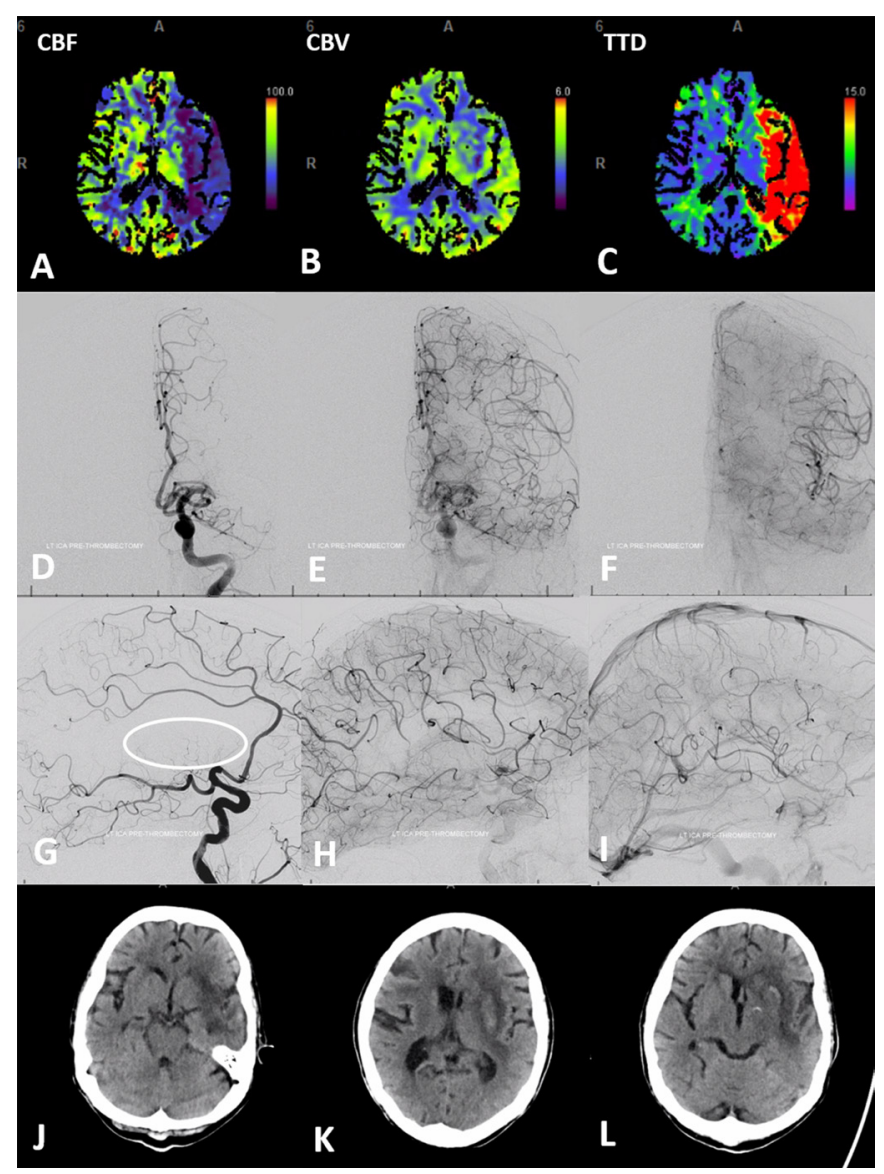

Figure 7 Classic pattern of leptomeningeal collateral support in the proximal middle cerebral artery (MCA) occlusion. Perfusion shows reduced $C B F(A)$, preserved $C B V(B)$, and increased time to drain (TTD) (C) corresponding with "penumbra" in the bulk of the MCA territory, with the exception of the lateral basal ganglia and the external capsule region. "Fetal" posterior cerebral artery (PCA) disposition allows for angiographic visualization of both the anterior cerebral artery (ACA)MCA and the PCA-MCA leptomeningeal collaterals in sequential frontal $(D, E, F)$ and lateral $(G, H, I)$ projections. Collateral response can be conceptualized as a shift in watershed territory, with operculum and insula being most vulnerable. $(\mathrm{J}, \mathrm{K}, \mathrm{L})$ Day 2 post-TICI (thrombolysis in cerebral infarction) 3 revascularization. CT reveals infarct of predicted lateral basal ganglia with petechial hemorrhagic transformation, and external capsule, as well as portions of the insula. Sparing of the caudate and globus pallidus is related to the dominance of the A1 segment lenticulostriate vessels (G, circle); unlike the patient in figure 6 . the upper convexity was preserved. CBF, cerebral flood flow; CBV, cerebral blood volume.

sense is one based on territory at risk. Despite significant differences, ASPECTS, ${ }^{20}$ CTA ASPECTS, ${ }^{21} 22$ and CT perfusion ${ }^{23-25}$ methods are all rooted in the concept of vascular territories. While the role of perfusion imaging in clinical decision-making remains a source of endless controversy, its diagnostic value in identifying the presence of a geographic hypoperfusion state and establishing the likely location of an occlusion (particularly for more "distal" occlusions) is invaluable (figures 6-9). A wellknown naming system by Gibo et $a l^{9}$ divides the MCA into 12 regions (figure 10 ). It is compatible with MCA variations and can be used in communicating both research and practice

scenarios.
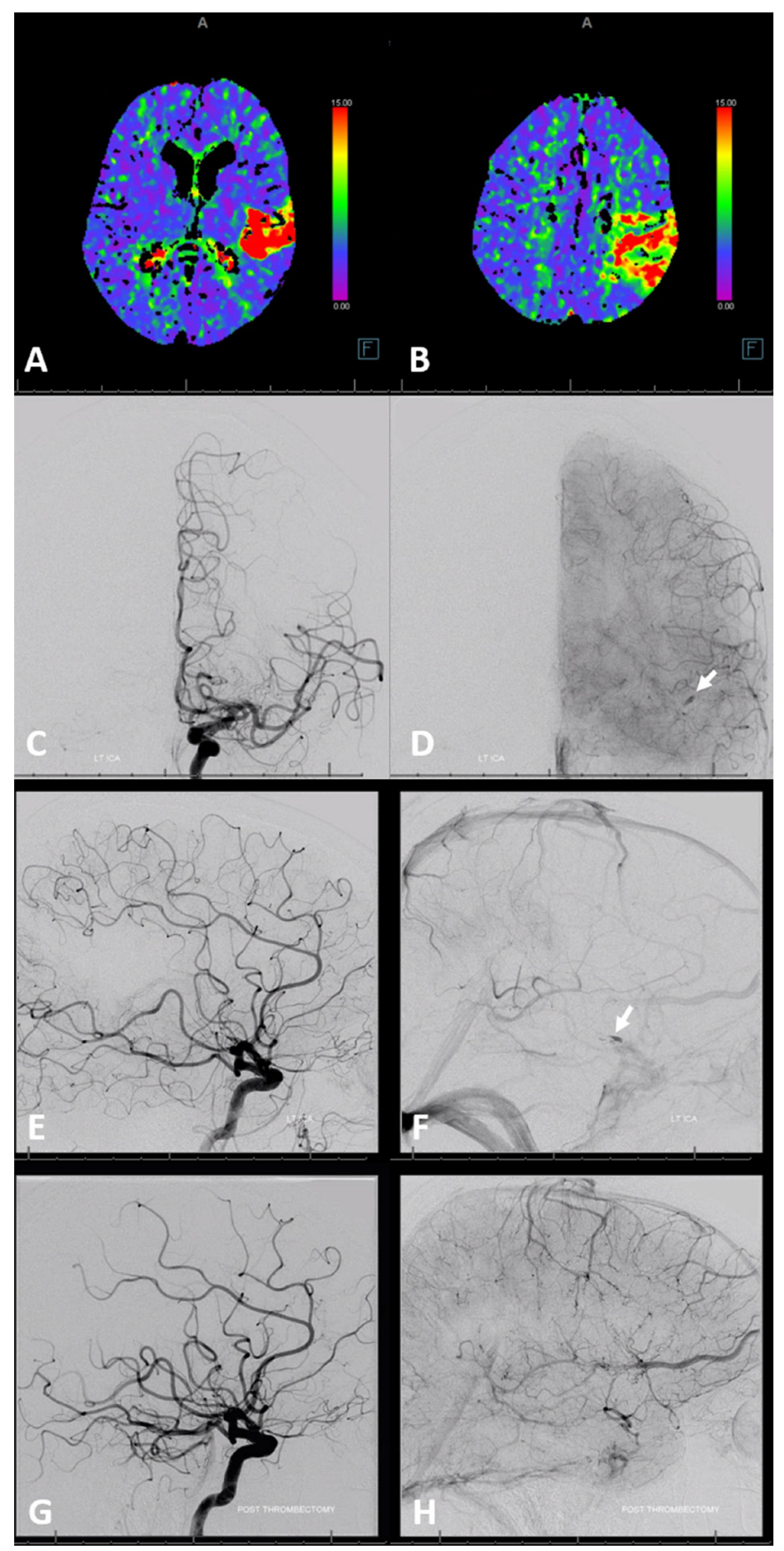

Figure 8 Life does not follow middle cerebral artery (MCA) branching pattern classifications. Mean transit time (MTT) maps show posterior temporal, parietal, and posterior frontal penumbra $(A, B)$. (C,D) shows an MCA "trifurcation". The embolus (arrows) lodged in the largest diameter straightest branch downstream of M1 segment flow vector. $(\mathrm{E}, \mathrm{F})$ Unlike classic "trifurcation" theory of each branch picking up frontal, parietal, and temporal lobes, this middle branch supplied both inferior temporal and rolandic areas in addition to entire parietal convexity-strategically producing a major set of clinical deficits despite a relatively modest volume at risk. $(\mathrm{G}, \mathrm{H})$ Post-thrombectomy.

\section{Hemodynamics and collateral circulation}

Hemodynamic factors are uniquely important from the perspective of thrombectomy. Emboli of sizes comparable to branch vessel inner diameters tend to lodge in the largest, straightest branches, with embolus lodging at a bifurcation point that prevents further migration (figures 6, 8 and 9). ${ }^{26} 27$ The same 


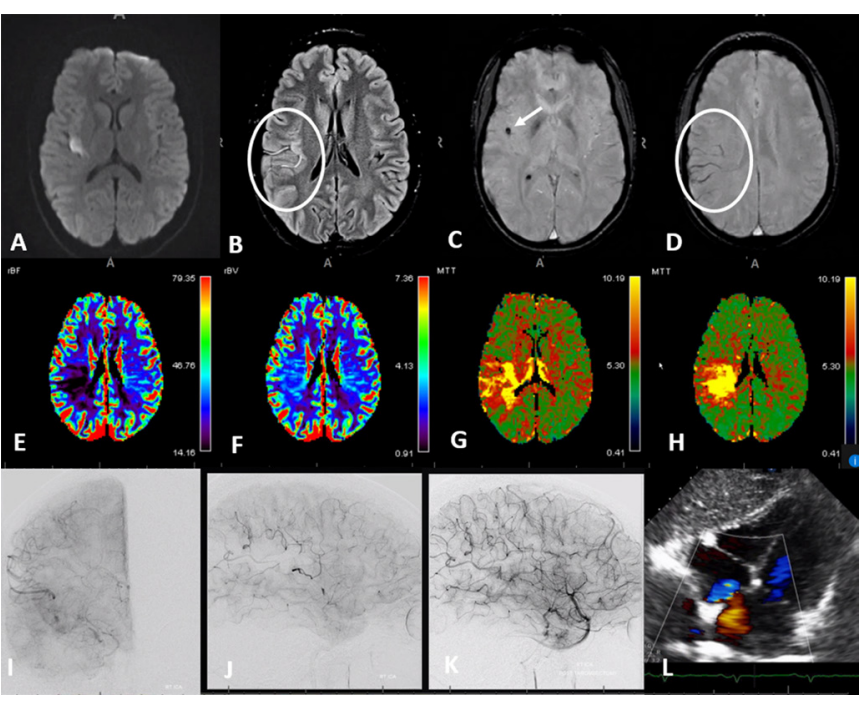

Figure 9 Advanced MR imaging of acute ischemia in a young patient with delayed presentation of the left upper extremity and facial weakness. Diffusion (A) and FLAIR (fluid attenuation inversion recovery) (B) showing slow flow in the distal fissure middle cerebral artery (MCA) branches. Susceptibility weighted imaging $(C, D)$ shows susceptibility in hemosiderin-rich embolus (arrow) and increased hemosiderin content in regional veins due to increased oxygen extraction (circle). MRI perfusion $(\mathrm{E}-\mathrm{H})$ shows decreased flow $(\mathrm{E})$, preserved volume $(\mathrm{F})$, increased mean transit time (MTT) (G), and increased delay $(\mathrm{H})$. Pre-thrombectomy angiography $(\mathrm{I}, \mathrm{J})$ shows occlusion of the parietal and rolandic territory (arrow=embolus). Post-thrombectomy (K). Large patent foramen ovale (PFO) (L).

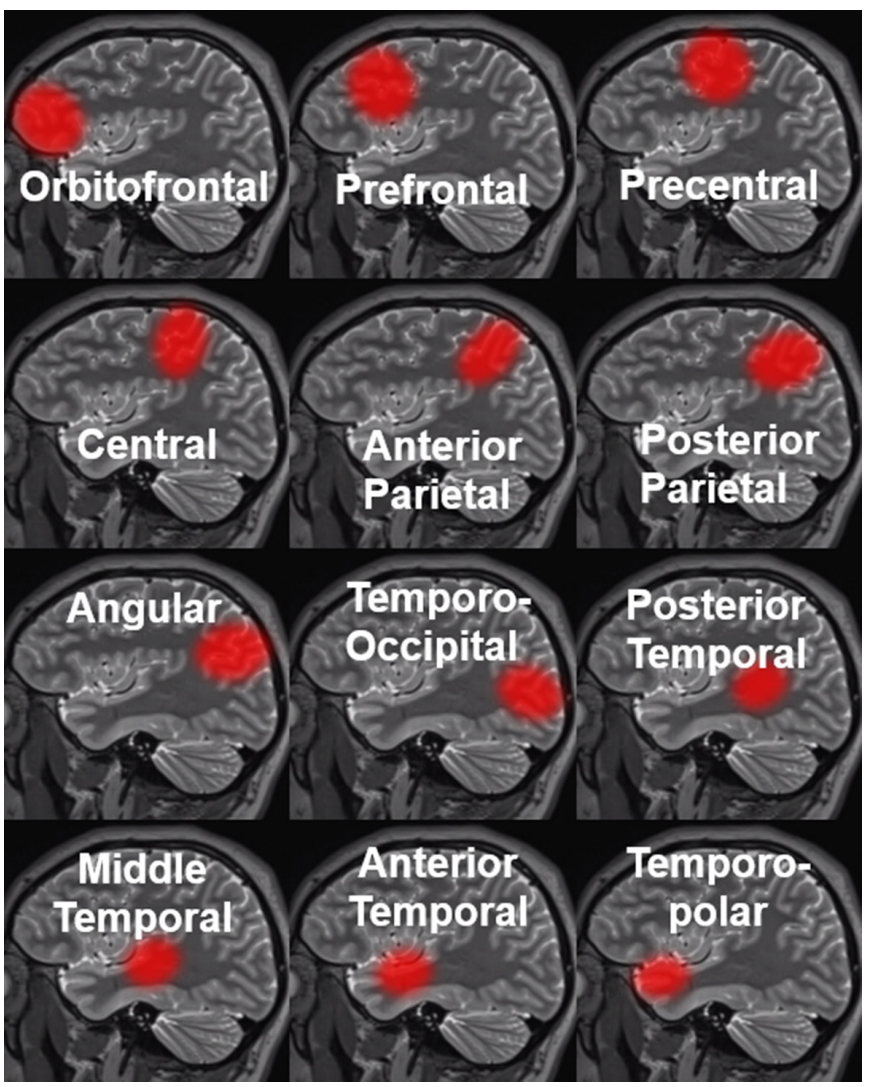

Figure 10 Classification of middle cerebral artery cortical territories according to Gibo et al. ${ }^{9}$ path, for different reasons, is also likely to be taken by a leading J-shaped microguidewire or by an aspiration catheter advanced by itself (the so-called "snake technique"). ${ }^{28}$ Tortuous MCA configuration is associated with reduced stent-triever thrombectomy efficacy. ${ }^{29}$ Similarly, the more acute cranial angulation of the anterior and mid-frontal superior division branches, combined with their relatively smaller sizes, make for more challenging "distal” thrombectomy targets. In contradistinction, vessels emerging more posteriorly from the Sylvian fissure (such as the temporo-occipital or parietal) tend to be straighter and usually somewhat larger in diameter, ${ }^{9}$ increasing the likelihood of both secondary embolization and successful distal thrombectomy (figures 8 and 9). High quality data for "distal" or "medium" vessel thrombectomy is currently lacking and significant challenges are anticipated in collecting enough of it. ${ }^{19}$

Efficiency of collateral support is the single most important factor determining brain tissue survival following acute primary route occlusion (figure 7 ). ${ }^{30}$ Beyond the circle of Willis, the collateral potential of cortical MCA territory (lenticulostriates have notoriously poor collateral support) is related to the efficiency of leptomeningeal ACA-MCA-PCA anastomoses. This efficiency is a complex product of genetic factors determining

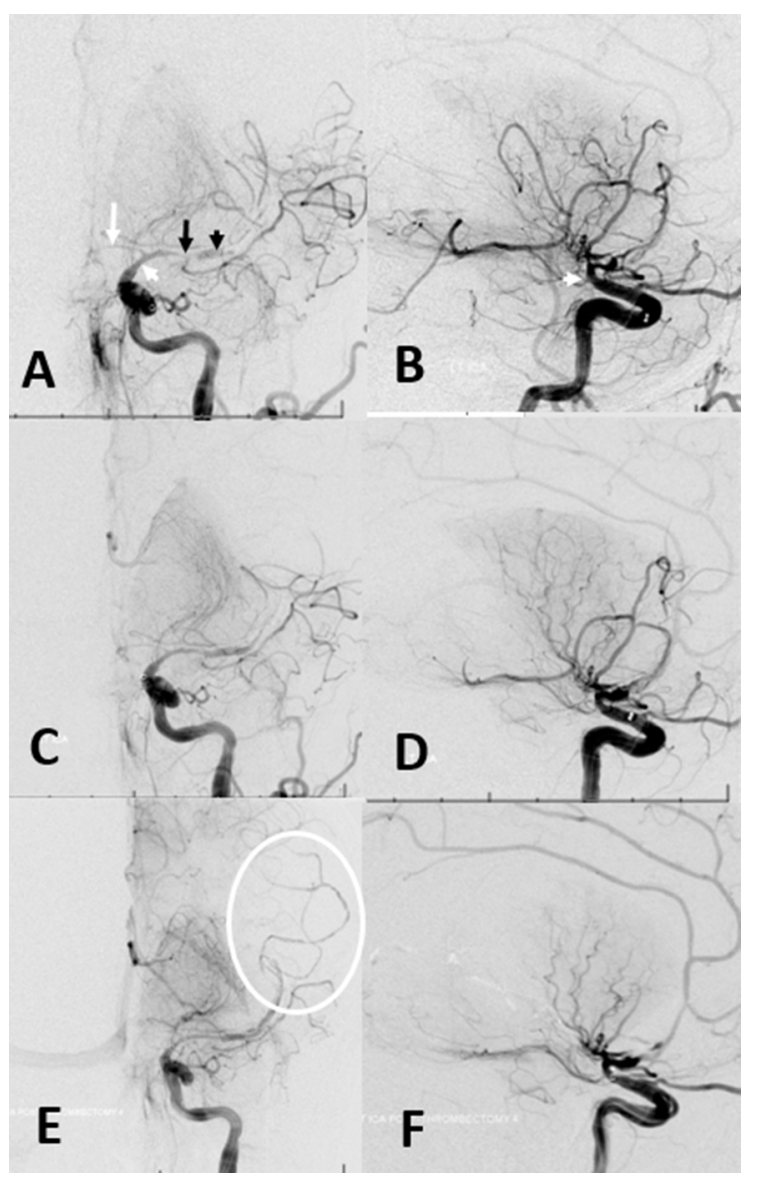

Figure 11 Acute intracranial dissection. (A,B) Initial images of supraclinoid stenosis (dissection origin, white arrowhead) extending into A1 (white arrow) and M1 (black arrow). A more "normal" caliber of the M2 segment (black arrowhead) with no distal vessels reflects contrast in the false lumen. $(C, D)$ Following attempted thrombectomy, an apparent "improvement" in proximal middle cerebral artery caliber reveals a corkscrew kind of M1/M2 tapering with no overall improvement in perfusion. $(E, F)$ Final images show corkscrew appearance of dissection extending into the $M 2 / M 3$ branches ( $E$, oval). 
intrinsic collateral potential and its modification by age, atheromatous disease, pre-existing ischemic states favoring collateral enhancement, and likely many other factors. ${ }^{31} 32$ Variation in individual collateral potential is simply astounding. Equally intriguing is the degree to which leptomeningeal collateral efficiency is enhanced by longstanding non-occlusive proximal flow constraints (such as intracranial atheromatous stenosis) in some patients, while similar lesions induce apparently little response in others.

Aside from the double-edge sword of induced or permissive hypertension, we have as of today no consistent way of enhancing collateral response in the acute setting. It seems that even highly effective leptomeningeal collaterals, producing initially low NIHSS scores, ultimately fail, favoring early intervention. ${ }^{32}$

\section{Non-embolic large vessel occlusive disease}

Entities such as large vessel atherosclerotic disease or intracranial dissection-a minority of large vessel occlusion etiologieslikely represent a substantial portion of modern thrombectomy "failures".

Atherosclerosis is a far less common cause of large vessel occlusion compared with emboli, even in susceptible populations. ${ }^{33} 34$ SAMMPRIS $^{35}$ (Stenting vs Aggressive Medical Management for Preventing Recurrent Stroke in Intracranial Stenosis) does not apply in the hyperacute setting. Prospective data on the safety and efficacy of acute revascularization in atheromatous occlusion is non-existent. ${ }^{36}$ Plaques can affect side branches (commonly lenticulostriates) and/or downstream territory. Only the latter is an appropriate present-day reperfusion target, with untoward occlusion of side branches (snowplow effect) being one of several major intervention hazards. ${ }^{35}$ Signs pointing to atheromatous (rather than embolic) disease include the presence of other stenoses, tapered vessel morphology proximal to occlusion, occlusion location not involving a major bifurcation and not along the main vector of flow, and-importantly-failure of durable reperfusion using well-established methods. Often, a small channel is repeatedly created, followed by repeat re-occlusion. Induction of full antiplatelet state and acute stenting are often required to maintain vessel patency. ${ }^{34} \mathrm{MCA}$ is a minority among this already small cohort, with more lesions involving basilar artery or carotid siphon. ${ }^{36}$

Intracranial dissection is a complex and controversial entity; evolution spans acute and delayed ischemic and hemorrhagic manifestations. ${ }^{37}$ In our experience, acutely occlusive MCArelated dissections usually present as a supraclinoid stenosis extending into M1 and A1 segments. Inadvertent propagation of dissection during reperfusion attempts is a distinct possibility. Unusual pruning of distal vessels, corkscrew morphology, and rapid re-occlusion without apparent concentric stenosis are some anatomical features (figure 11). Outcomes seem unpredictable, and more related to collateral efficiency rather than success of reperfusion.

\section{CONCLUSIONS}

Appreciation of and comfort with variation, which is the rule when it comes to the MCA, is possible through knowledge of practical embryology. A pragmatic approach of understanding vascular anatomy based on parenchymal territories target vessels supply rather than by their sites of origin is invaluable during thrombectomy. Interpretation of advanced imaging often helps identify both the location and the extent of at-risk territory, and suggests the level of occlusion, especially for more distal emboli.

Contributors All of the following authors, MS, ER, EN, BC, KI, PKN, have made:
Substantial contributions to the conception or design of the work; or the acquisition, analysis, or interpretation of data for the work; AND Drafting the work or revising it critically for important intellectual content; AND Final approval of the version to be published; AND All in agreement to be accountable for all aspects of the work in ensuring that questions related to the accuracy or integrity of any part of the work are appropriately investigated and resolved. All authors meet criteria for substantial contributions to this manuscript.

Funding The authors have not declared a specific grant for this research from any funding agency in the public, commercial or not-for-profit sectors.

Competing interests None declared.

Patient consent for publication Not required.

Provenance and peer review Commissioned; internally peer reviewed.

\section{REFERENCES}

1 Lasjaunias PL, Berenstein A, KGt B. Surgical neuroangiography. 2nd ed. Berlin, New York: Springer, 2001.

2 El Falougy $H$, Selmeciova P, Kubikova E, et al. The variable origin of the recurrent artery of Heubner: an anatomical and morphometric study. Biomed Res Int 2013;2013:873434

3 Gomes F, Dujovny M, Umansky F, et al. Microsurgical anatomy of the recurrent artery of Heubner. J Neurosurg 1984;60:130-9.

4 Teal JS, Rumbaugh CL, Bergeron RT, et al. Anomalies of the middle cerebral artery: accessory artery, duplication, and early bifurcation. Am J Roentgenol Radium Ther Nucl Med 1973;118:567-75.

5 Abanou A, Lasjaunias P, Manelfe C, et al. The accessory middle cerebral artery (AMCA). diagnostic and therapeutic consequences. Anat Clin 1984;6:305-9.

6 Chang HY, Kim MS. Middle cerebral artery duplication : classification and clinical implications. J Korean Neurosurg Soc 2011;49:102-6.

7 Komiyama M, Nakajima $\mathrm{H}$, Nishikawa $\mathrm{M}$, et al. Middle cerebral artery variations: duplicated and accessory arteries. AJNR Am J Neuroradiol 1998;19:45-9.

8 Gailloud P, Albayram S, Fasel JHD, et al. Angiographic and embryologic considerations in five cases of middle cerebral artery fenestration. AJNR Am J Neuroradiol 2002;23:585-7.

9 Gibo H, Carver CC, Rhoton AL, et al. Microsurgical anatomy of the middle cerebral artery. J Neurosurg 1981;54:151-69.

10 Tanriover N, Kawashima M, Rhoton AL, et al. Microsurgical anatomy of the early branches of the middle cerebral artery: morphometric analysis and classification with angiographic correlation. J Neurosurg 2003;98:1277-90.

11 Umansky F, Juarez SM, Dujovny M, et al. Microsurgical anatomy of the proximal segments of the middle cerebral artery. J Neurosurg 1984;61:458-67.

12 Goyal M, Menon BK, Krings T, et al. What constitutes the M1 segment of the middle cerebral artery? J Neurointerv Surg 2016;8:1273-7

13 Lobsien D, Gawlitza M, Hoffmann K-T, et al. Comment on the article what constitutes the M1 segment of the middle cerebral artery? J Neurointerv Surg 2017;9:524.

14 Saber H, Narayanan S, Palla M, et al. Mechanical thrombectomy for acute ischemic stroke with occlusion of the M2 segment of the middle cerebral artery: a metaanalysis. J Neurointerv Surg 2018;10:620-4.

15 Kunz WG, Almekhlafi MA, Goyal M. Distal vessel occlusions: when to consider endovascular thrombectomy. Stroke 2018;49:1581-3.

$16 \mathrm{Kim} \mathrm{CH}$, Kim S-E, Jeon JP. Meta-analysis of endovascular treatment for acute M2 occlusion. J Korean Neurosurg Soc 2019;62:193-200.

17 Seker F, Pfaff J, Neuberger U, et al. Comparison of superior and inferior division occlusions treated with endovascular thrombectomy. Clin Neuroradiol 2019;387. doi:10.1007/s00062-019-00767-3. [Epub ahead of print: 20 Feb 2019].

18 Rai AT, Domico JR, Buseman C, et al. A population-based incidence of M2 strokes indicates potential expansion of large vessel occlusions amenable to endovascular therapy. J Neurointerv Surg 2018;10:510-5.

19 Grossberg JA, Rebello LC, Haussen DC, et al. Beyond large vessel occlusion strokes: distal occlusion thrombectomy. Stroke 2018;49:1662-8.

20 Pexman JH, Barber PA, Hill MD, et al. Use of the Alberta stroke program early CT score (ASPECTS) for assessing CT scans in patients with acute stroke. AJNR Am J Neuroradiol 2001:22:1534-42.

21 Sallustio F, Motta C, Pizzuto S, et al. CT angiography aspects predicts outcome much better than noncontrast $\mathrm{CT}$ in patients with stroke treated endovascularly. AJNR Am J Neuroradiol 2017:38:1569-73.

22 Bhatia R, Bal SS, Shobha N, et al. CT angiographic source images predict outcome and final infarct volume better than noncontrast $\mathrm{CT}$ in proximal vascular occlusions. Stroke 2011:42:1575-80.

23 Albers GW, Marks MP, Kemp S, et al. Thrombectomy for stroke at 6 to 16 hours with selection by perfusion imaging. N Engl J Med 2018;378:708-18.

24 Austein F, Riedel C, Kerby T, et al. Comparison of perfusion CT software to predict the final infarct volume after thrombectomy. Stroke 2016;47:2311-7.

25 Lansberg MG, Christensen S, Kemp S, et al. Computed tomographic perfusion to predict response to recanalization in ischemic stroke. Ann Neurol 2017;81:849-56.

26 Bushi D, Grad Y, Einav S, et al. Hemodynamic evaluation of embolic trajectory in an arterial bifurcation: an in-vitro experimental model. Stroke 2005;36:2696-700. 
27 Chung EML, Hague JP, Chanrion M-A, et al. Embolus trajectory through a physical replica of the major cerebral arteries. Stroke 2010;41:647-52.

28 Heit JJ, Wong JH, Mofaff AM, et al. Sofia intermediate catheter and the snake technique: safety and efficacy of the Sofia catheter without guidewire or microcatheter construct. J Neurointerv Surg 2018;10:401-6.

29 Schwaiger BJ, Gersing AS, Zimmer C, et al. The curved MCA: influence of vessel anatomy on recanalization results of mechanical thrombectomy after acute ischemic stroke. AJNR Am J Neuroradiol 2015;36:971-6.

30 Nogueira RG, Jadhav AP, Haussen DC, et al. Thrombectomy 6 to 24 hours after stroke with a mismatch between deficit and infarct. N Engl J Med 2018;378:11-21.

31 Piedade GS, Schirmer CM, Goren 0, et al. Cerebral collateral circulation: a review in the context of ischemic stroke and mechanical thrombectomy. World Neurosurg 2019;122:33-42.
32 Bang OY, Saver JL, Kim SJ, et al. Collateral flow predicts response to endovascular therapy for acute ischemic stroke. Stroke 2011;42:693-9.

33 Turin TC, Kita Y, Rumana N, et al. Ischemic stroke subtypes in a Japanese population: Takashima Stroke Registry, 1988-2004. Stroke 2010:41:1871-6.

34 Chang Y, Kim BM, Bang OY, et al. Rescue stenting for failed mechanical thrombectomy in acute ischemic stroke: a multicenter experience. Stroke 2018;49:958-64.

35 Chimowitz MI, Lynn MJ, Derdeyn CP, et al. Stenting versus aggressive medical therapy for intracranial arterial stenosis. N Eng/ J Med 2011;365:993-1003.

36 Zhou T, Li T, Zhu L, et al. Intracranial stenting as a rescue therapy for acute ischemic stroke after Stentriever thrombectomy failure. World Neurosurg 2018;120:e181-7.

37 Krings T, Choi I-S. The many faces of intracranial arterial dissections. Interv Neuroradiol 2010;16:151-60. 